\title{
An Overview of the Teaching Assistant Consultant Program for Developing Competency in Novice Engineering Graduate Teaching Assistants
}

\author{
Kush Bubbar ${ }^{1}$, Alexandros Dimopoulos ${ }^{1}$, Cynthia Korpan ${ }^{2}$, Peter Wild ${ }^{1}$ \\ ${ }^{1}$ Faculty of Engineering, University of Victoria, BC, Canada \\ ${ }^{2}$ Learning and Teaching Centre, University of Victoria, BC, Canada \\ Corresponding Author: kbubbar@uvic.ca
}

\begin{abstract}
As engineering education strives to progress towards a student-centric learning model, a competency gap with future educators becomes more apparent. In particular, the expectation of graduate student teaching assistants (GTAs) in attaining teaching competency to support this dynamic learning environment, often without sufficient training, is unrealistic.

In the following paper, we present an implementation of the flexible Teaching Assistant Consultant (TAC) program, which serves to support the development of novice GTA competencies using a discipline-specific model with emphasis on assisting the unique challenges of international teaching assistants.

We introduce the specific role of the TAC in terms of core principles and deliverables, and the strategic structure of the campus wide program at the University of Victoria. We conclude by detailing the specific implementation of the program in engineering by illustrating the role and deliverables of the engineering TAC.
\end{abstract}

Keywords: Graduate Teaching Assistant Training: Teaching Assistant Consultant Program: Skills-based Workshops: Community of Practice: International Teaching Assistant Training

\section{INTRODUCTION}

As Canadian universities continue to drift towards a student-centred learning environment, the role of the Graduate Teaching Assistant (GTA) continues to evolve [1]. In the modern university, the expectation of the graduate student is concurrent exceptionalism as a teacher and researcher, which is a significant departure from the original sole requirement of research excellence [1]. As such, teaching excellence is regarded as a competency gap with novice GTAs, and has initiated corresponding interest in research [2].

In particular, skills based Teaching Assistant (TA) training programs are viewed as an effective approach to develop competence in novice GTAs. Research on identifying the learning outcomes for novice GTAs plays an important role in defining the scope of these training programs. For example, Boman [2] and Meadows [3], through their research on TA induction programs and international TAs respectively, have identified a consolidated list of three top level learning outcomes for their TA training programming:

1. Improving Teacher Self-efficacy

2. Emphasizing Student-Centred Teaching

3. Decreasing Anxiety

Self-efficacy, in the context of teaching, refers to the conviction that a GTA can successfully perform the required teaching behaviours to produce the desired outcome (i.e. self-confidence the GTA can teach effectively) [2]. Research has shown teachers with a high sense of self-efficacy are more likely to be applying effective teaching practices, coupled with increased levels of enthusiasm, organization, and planning [3].

Student-centred teaching places the student at the centre of the learning process by promoting both active learning and inquiry based learning techniques. In this environment, students question and apply their knowledge to complete tasks, resulting in a deeper understanding of the material [3].

A fear of public speaking often invokes anxiety in novice GTAs. This is an issue in STEM education, as GTAs are often required to teach tutorials and laboratories. Research has shown, this anxiety can be reduced through skills-based training programs [4].

\subsection{Institutional Level TA Training}

To support the realization of these outcomes, efforts have been put forth to define the associated TA competencies [5]. Most institutions have implemented centralized TA training programs through their respective 
learning and teaching centres, thus providing a mechanism to address the outstanding skills gap [6], [7].

Institutional level training programs often provide a one-time, university wide training to all novice GTAs by delivering instruction on topics such as generic teaching approaches, ethics, and campus resources through a combination of lectures and a skills-based workshop model [7]. While these programs deliver important training material, and are efficient with resource allocation, they cannot support discipline-specific training needs regarding knowledge, culture, and experience.

\subsection{Argument for Department Level TA Training}

Most departments on a university campus operate as a silo, with their own unique procedures and practices, often tightly tied to historical evolution. Becoming a successful GTA requires an understanding of how to traverse the unique culture of a particular department.

Conversely, intrinsic to each field of scholarly work, there exist discipline-specific signature pedagogies, which may not be relevant to alternative fields of study. For example, organic chemistry is taught with students interacting with physical kits in which spherical balls and cylindrical pegs represent atoms and covalent bonds. Consequently, this content is domain specific to chemistry students and, thus, is outside the scope of an institutional level training program.

Finally, to accelerate the rate at which novice GTAs acquire competence, GTAs require ongoing training with discipline-specific feedback. Such a program is challenging to both support various levels of competence, while scaled to operate at the institution level.

There are thus multiple motivations for implementing components of novice GTA training at the departmental level [7].

\subsection{Unique Training Needs of International TAs}

International Teaching Assistants (ITAs) constitute a significant percentage of new TAs at Canadian institutions [8]. In the STEM disciplines, the majority of ITAs come from high power distance cultures (e.g. Iran, India, and China), where the status between instructor and student is large [3]. Education in these environments is primarily teacher-centric and contrasts the student-centric learning environments at Canadian universities. In addition, novice ITAs are often teaching in a second language where the norms and expectations conflict with those from their home cultures, leading to more anxiety and lower self-efficacy [3]. As such, novice ITAs are often operating in "survival mode" and thus are more teachercentric in their orientation. As discussed by Meadows et al., ITAs benefit from additional targeted training on cultural differences and communication styles [3].

The motivation of this paper is to introduce the Teaching Assistant Consultant (TAC) program to the engineering community, as implemented at University of Victoria (UVic), as a basis to address both the disciplinespecific and ITA competency challenges of novice GTAs.

\section{TEACHING ASSISTANT TRAINING PROGRAMS AT UVIC}

At (UVic), most TA training programs are centralized through the Learning and Teaching Centre (LTC). This includes institution wide workshops offered throughout the calendar year, as well as, a TA conference held at the start of each academic semester. These training events serve to deliver generic and faculty-specific content applicable to all GTAs across campus, and is in line with the structure at most academic institutions across Canada [6]. A unique department-based training structure, as viewed in Fig. 1, is the internal interface between the LTC and most departments on campus via the Teaching Assistant Consultant (TAC) program.

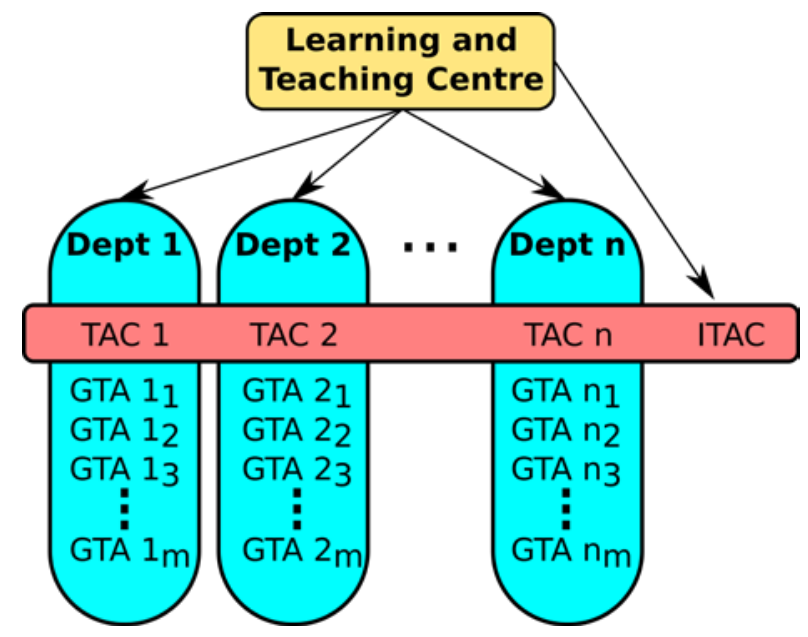

Fig. 1. TA Training Structure at UVic. $\mathrm{n} \equiv$ department number, $\mathrm{m} \equiv$ number of GTAs

\subsection{What is a Teaching Assistant Consultant?}

The TAC program has its origins at the University of California [9], [10] with a mission to improve undergraduate education by: 1) working directly with instructors to strengthen teaching practices; 2) providing professional development opportunities for GTAs, 3) 
fostering a positive community of practice where discussions about teaching are encouraged.

In general, TACs are senior graduate students with teaching assistant (TA) experience and demonstrated teaching excellence whom play the role of a teaching mentor [11]. They fulfil this role through delivering experiential-based workshops to enhance teaching effectiveness in addition to offering one-on-one consultations to GTAs to cater to individual training needs [9], [11].

Coming to UVic from UCLA, Teresa Dawson worked collaboratively with Cynthia Korpan to implement the TAC program with a subtle but important variation. Their program used a shared employment model between the LTC and the target department [12]. In this revised structure, both parties have invested interests in the TAC deliverables. The TAC, whom is already an experienced GTA in their home department, is tasked to collaborate with department leadership to help define key GTA challenges, and implement appropriate training to address these challenges [11], [13]. In utilizing such a model, the LTC effectively gains access inside the silo walls of each university department and positively influences the value of teaching to their respective GTAs.

\subsection{TAC Program Structure}

The mandate of the TAC program is to support the professional development of GTAs through guided mentorship. TACs achieve this mandate, by adhering to a program structure detailed in the TAC guidebook [14]. The following section summarizes the main components of the guidebook.

2.2.1 Quality and Sustainability. Two fundamental principles of the TAC program are: 1) continuous quality improvement, and 2) program sustainability [11], [14]. Continuous quality improvement is reinforced through gathering feedback. Questionnaires are administered pre and post workshop delivery and at the program level. Data compilation helps determine the effectiveness of the teaching methods employed. Each TAC addresses program sustainability by articulating their progress and recommendations through a detailed final report. This report is shared with the successive TAC, and serves as a historical reference.

2.2.2 Core Deliverables. The TAC role comprises delivering a set of core outputs. These outputs include: 1 ) developing and delivering eight hours of teaching related instruction via experiential workshops; 2) providing oneon-one consultation hours for GTAs in their home department; 3) conducting teaching observations of GTAs and providing feedback; 4) supporting GTAs in developing a written reflection piece on their professional development experiences; 5) supporting GTAs in developing an original instructional material; 6) generating a final report which articulates accomplishments and recommendations; and 7) fostering a TA community where GTAs have a safe place to discuss their challenges and successes.

2.2.3 Training the Trainer. To establish a common level of competency amongst TACs, a four-day experientialbased training session is administered by the LTC to all TACs at the start of their appointment, for which basic instruction is delivered on: 1) learning outcomes and lecture design, 2) microteaching, teaching observations, and delivering effective feedback, 3) effective strategies for performing one-on-one consultations, and 4) introduction to various campus resources via guest speakers. This TAC retreat serves the additional task of creating a Community of Practice (CoP), for which TACs are encouraged to support one another in developing and delivering training material.

2.2.4 International Teaching Assistant Consultant. Unique to UVic is the creation of the International Teaching Assistant Consultant (ITAC) position filled by an ITA with the same significant teaching qualifications required by all TACs. The ITAC is responsible for supporting the specific needs of ITAs detailed in Section 1 , but does not have a home department. The ITAC is responsible for delivering instruction via the LTC at the institution level, and is a resource each individual TAC may use in delivering specific training to the ITAs in their respective home departments.

\section{ENGINEERING TAC ROLE AT UVIC}

Section 1 articulated the discipline-specific and ITA competency gap with novice GTAs, while Section 2 introduced UVic's implementation of the TAC program. The following section presents a brief overview of the TAC program as implemented at the engineering faculty level.

\subsection{Identifying Engineering GTA Learning Outcomes}

Using historical TAC reports in conjunction with multiple consultations between the department chairs and graduate advisors, the engineering TAC identified the following learning outcomes for novice GTAs:

- $\quad$ Professionalism of GTAs

- $\quad$ TA role expectation in engineering 
- Promoting diversity in the classroom

- Knowledge of campus resources for ITAs

- Planning and time management

- Managing conflict with students

- Fostering a self-guided learning environment

The engineering TACs, have over time, addressed each of these learning outcomes in some capacity through their program development. The following sections briefly detail the current program implementation.

\subsection{Workshops}

As discussed in Section 2.2.2, TACs are responsible for delivering at least eight hours of experiential training via discipline-specific workshops. To date, five discipline-specific workshops have been developed and delivered to the engineering GTA body.

3.2.1 Being an International TA at UVic. As mentioned in Section 1.2, most ITAs benefit from targeted training on cultural differences and communication styles. The ITAC presents this workshop from the lens of an experienced and successful ITA, by introducing new ITAs to the Canadian university culture, presenting dress standards, body language, formal versus informal communication, and customary levels of authoritative behaviour. This workshop is mandatory for all new ITAs.

3.2.2 Roles and Responsibilities of an Engineering TA. This workshop introduces new GTAs to the different roles and responsibilities of engineering TAs at UVic (i.e. marker, tutorial, lab, and design). Key points on professionalism are presented as a checklist, followed by introducing the initiative of promoting diversity. Practical TA tips are offered and GTAs are made aware of additional teaching resources available on campus. The workshop concludes with a video of an undergraduate student speaking about their experience with both a "good" and "bad" TA, and GTAs are tasked with identifying both positive and negative attributes. This workshop is mandatory for all new GTAs.

3.2.3 Time Management. The goal of this workshop is to: 1) help GTAs comprehend the concept of time in the context of their daily lives, and 2) provide pragmatic methods to develop feasible plans. The former goal is achieved by introducing the concept of mapping an individual's physical and mental energy level for a typical day. Once completed, the GTA contrasts their activities with their energy level to determine their effectiveness (e.g. exercising when mental energy is low and physical energy is high is an effective use of time). The intent of this exercise is to be informative and non-judgemental.
For the latter goal, a hybrid of the SMART (Specific, Measureable, Attainable, Relevant, Time-bound) goal definition and Kanban operational frameworks are presented as a basis for generating a simple project timeline. GTAs follow with an exercise by building their own project plan, in the context of GTA deliverables, under the guidance of the engineering TAC.

3.2.4 Conflict Management. This workshop presents conflict in the context of Rosenberg's non-violent communication [15]. Conflict is discussed both broadly and from the perspective of GTAs and students. Through strategic prompting, the engineering TAC helps the GTAs identify the ambiguity of student marking as a primary source of conflict between students and GTAs, and an exercise to reduce conflict through the application of a rubric in marking is undertaken. A second part of the workshop focuses on unavoidable conflict and presents the LEAP framework (Listen, Empathize, Ask Questions, and Propose) as a mindful and supportive approach to address the conflict. The workshop concludes with GTAs practicing using the LEAP framework during several roleplaying scenarios.

3.2.5 Coaching and Facilitation. This workshop introduces GTAs to the profession of coaching and defines the role of a coach as fostering self-guided learning in a student-centric learning environment. Stem sentences, as well-crafted inquiry driven questions, are introduced, and exemplified through role-playing activities. Students are encouraged to create their own discipline-specific stem sentences. A licensed PCC [16] (Professional Certified Coach) facilitates this workshop. Further details of this workshop are found in both Bubbar et al. [17] and Dimopoulos et al. [18].

\subsection{Building a Community of Practice}

For supporting the ongoing professional training needs of GTAs, GTAs are encouraged to follow up with the engineering TAC via open discussions in a social context (e.g. GTA coffee time). Doing so, serves to foster a sense of community amongst the engineering GTAs and generates more motivation to further refine their skills [7].

\subsection{One-on-One Consultation}

For circumstances where a GTA seeks confidential support, the engineering TAC offers one-on-one consultation. This service is available for a variety of situations ranging from advice on how to select an appropriate TA position, to specific challenges a GTA is facing in a classroom environment, or even a request for a live teaching observation. Where appropriate, the TAC is 
instructed to refer to professional campus resources such as counselling services.

\subsection{Central Access Point for all GTA Content}

The Moodle Learning Management System is used to facilitate a centralized repository of information regarding training content and campus resources, while simultaneously providing a mechanism for all GTAs to communicate via social forums.

\section{CONCLUSION}

The intention of this work is to introduce UVic's implementation of the Teaching Assistant Consultant program to the engineering community, as a basis for addressing discipline-specific and ITA competency gaps for novice GTAs. We believe this paper has met this intention, however we are always keen on soliciting constructive feedback. One of our core program goals is sharing the model, so please contact us if you would like more information [14].

\section{Acknowledgements}

The authors graciously acknowledge support by the Learning and Teaching Centre at the University of Victoria for their time and commitment in developing this innovative program. We also appreciate the financial support from Natural Sciences and Engineering Research Council (NSERC) of Canada Design Chair for funding this publication.

\section{References}

[1] A. E. Austin and D. H. Wulff, "The challenge to prepare the next generation of faculty," Paths Profr. Strateg. Enrich. Prep. Future Fac., pp. 3-16, 2004.

[2] J. S. Boman, "Graduate Student Teaching Development: Evaluating the Effectiveness of Training in Relation to Graduate Student Characteristics," Can. J. High. Educ. Tor., vol. 43, no. 1, pp. 100-114, 2013.

[3] K. N. Meadows, K. C. Olsen, N. Dimitrov, and D. L. Dawson, "Evaluating the Differential Impact of Teaching Assistant Training Programs on International Graduate Student Teaching," Can. J. High. Educ. Tor., vol. 45, no. 3, pp. 34-55, 2015.

[4] C. B. Pribyl, J. Keaten, and M. Sakamoto, "The effectiveness of a skills-based program in reducing public speaking anxiety,” Jpn. Psychol. Res., vol. 43, no. 3, pp. 148-155, Sep. 2001.

[5] C. Korpan, S. L.-M. Sheffield, and R. Verwoord, "Teaching Assistant Competencies in Canada:
Building a Framework for Practice Together," Collect. Essays Learn. Teach., vol. 8, pp. 219-230, 2015.

[6] C. Korpan and D. Murray, "Preparing Teaching Assistants (TAs): A National Survey of Canadian Post-secondary Institutions' TA Orientations," Teaching Assistant and Grad Student Advancement (TAGSA), Nov. 2014.

[7] R. L. Kajfez and H. M. Matusovich, "Competence, Autonomy, and Relatedness as Motivators of Graduate Teaching Assistants,” J. Eng. Educ., vol. 106, no. 2, pp. 245-272, Apr. 2017.

[8] N. LeGros and F. Faez, "The intersection between intercultural competence and teaching behaviors: A case of international teaching assistants.," J. Excell. Coll. Teach., vol. 23, no. 3, 2012.

[9] “UC Davis Teaching Assistant Consulting Program," TAC Program at UC Davis, 02-May-2017. [Online]. Available: https://cee.ucdavis.edu/teachingsupport/become-tac.html. [Accessed: 02-May-2017].

[10] "Departmental TA Training and the TAC Program," TAC Program at UCLA, 02-May-2017. [Online]. Available: http://www.oid.ucla.edu/training/ta. [Accessed: 02-May-2017].

[11] C. Korpan, "Conversations and Collaborations," Collect. Essays Learn. Teach., vol. 3, pp. 83-88, 2010.

[12] T. Dawson, "Quick Chat with Kush Bubbar RE: Research Resources for TAC Program,” 04-May2017.

[13] T. Dawson and L. Larry, "Integrating Technology into Undergraduate Teaching: Developing a Model of Advanced TA Consultants in the Disciplines,” University of California—Los Angeles, Final Report for the Fund for the Improvement of Post-Secondary Education (FIPSE) grant \#P116B71855, 2000.

[14] C. Korpan, "Teaching Assistant Consultant (TAC) Guidebook 2015-2016,” University of Victoria, Learning and Teaching Centre, Jun. 2015.

[15] M. B. Rosenberg, Nonviolent communication: A language of compassion. PuddleDancer Press Del Mar, CA, 1999.

[16] "Professional Certified Coach (PCC) - Individual Credentialing - ICF.” [Online]. Available: https://coachfederation.org/credential/landing.cfm?Ite mNumber=2202. [Accessed: 08-May-2017].

[17] K. Bubbar, A. Dimopolous, R. Gaetz, P. Wild, and M. McWilliam, "Evolution of the Design Engineering Mentorship Program," in Proceedings of the Canadian Engineering Education Association, Halifax, Nova Scotia, Canada, 2016.

[18] A. Dimopoulos, K. Bubbar, R. Gaetz, and P. Wild, "Training Teaching Assistants as Coaches," in Proceedings of the Canadian Engineering Education Association, Toronto, Ontario, Canada, 2017. 